\title{
PERFORMANCE MODELLING AND ANALYSIS OF PASSIVE OPTICAL NETWORKS FOR POISSON TRAFFIC
}

Passive Optical Networks (PONs) are the most important class of fiber-based access systems, capable of delivering services with numerous applications, such as high-definition video and video conferencing. In this paper we perform call-level performance analysis of the upstream direction in TDMA-based PONs and hybrid TDMA-WDM PONs. The proposed analysis is based on well-established recursive teletraffic models and leads to Call Blocking Probabilities determination. We consider the case where the optical access network accommodates multiple serviceclasses with Poisson arriving calls. The accuracy of the proposed analytical models is validated by simulation and is found to be absolutely satisfactory.

\section{Introduction}

The information and application explosion that we are currently experiencing have forced the research community to the development of optical access networks, since advantages in the optical technology significantly reduced the cost of introducing fiber to the local loop. An access network is generally known as the "first mile" network that connects service providers with small businesses and residential users. The users of access networks demand a cost-effective access at high data rates and with Quality of Service (QoS) guarantees. Similarly, businesses request a broadband infrastructure, where their local networks can be efficiently connected to the Internet. In addition, service providers wish for the development of reliable and scalable access solutions with the perspective of adjusting to future applications and to the growing bandwidth needs.

Over the past several years telecom industries focused on the development of high capacity backbone networks. In contrast, existent access solutions, such as Digital Subscriber Line (DSL), provide relatively low data rates at the downstream channel (a few Mbps) and at the upstream channel (a few hundreds of Kbps) [1]. Therefore, access networks are considered as the basic drawback for providing broadband services, such as video on-demand, interactive games, video conference, etc.

Currently, the dominant broadband access solutions are DSL and Community Antenna Television (CATV). These technologies report several disadvantages, since they are based on infrastructures, originally developed for carrying voice traffic only. In DSL, an important constraint is the distance between end users and service providers, which cannot exceed $6 \mathrm{Km}$, due to the signal attenuation [1]. Therefore only $60 \%$ of residential subscribers may access the Internet through a DSL [2]. Recently, variations of DSL are introduced, such as Very high bit-rate DSL (VDSL) which can support up to $50 \mathrm{Mbps}$ of downstream bandwidth, but with more severe distance limitations ( $~ 500 \mathrm{~m})$. On the other hand, CATV networks can offer Internet access by reserving Radio Frequency (RF) channels in coaxial cables. Since cable networks were originally built for delivering broadcast services, they have a poor performance when they provide access bandwidth.

The abovementioned problems of the existent access networks can be confronted with the introduction of optical technology, given that optical fibers provide huge capacity with very low losses. Optical access networks can support data rates of several Gbps, while their cost is similar to that of DSL [3]. Since optical fibers can support any kind of traffic format, the installation of optical fibers to access networks is an investment, providing insurance for supporting future applications. Moreover, the installation of optical fibers must meet economic parameters, since in many cases service providers wish to maintain their investments in the existent infrastructure. $85 \%$ of the total cost of a new optical access network refers to civil works, while only $3 \%$ refer to the equipment cost [4]. Therefore, in cases of installing a new network, the optical fiber is installed up to the subscriber's location (Fiber-To-The-Home, FTTH), or if it is desirable to maintain parts of the existing network, fibers are installed up to the curb (Fiber-To-The-Curb, FTTC), or up to the building (Fiber-To-The-Building, FTTB) [4].

Different fiber-based access architectures have been proposed (or even deployed in some countries) [4]. The implementation of point-to-point access architectures by using dedicated fibers to each subscriber offers huge bandwidth to the subscriber; however, the cost of the users' equipment is high, since a pair of transmitters has to be installed for each point-to-point connection. An alternate solution that offers shared access refers to the installation of an active node in the users' premises. This approach reduces

\footnotetext{
* J. S. Vardakas, V. G Vassilakis, M. D. Logothetis

Wire Communications Laboratory, Department of Electrical \&Computer Engineering, University of Patras, Greece.

Email: jvardakas@wcl.ee.upatras.gr
} 
the users' equipment cost, but maintenance and power supply to the active node is required. Another shared access architecture is the Passive Optical Network (PON), where the active node is replaced with a passive device. PONs have several advantages over active node architectures. PONs incur lower capital expenditures, since no electronic components are used. The overall cost is further decreased by the absence of power supplying and maintenance of the passive node [5]. Moreover, PONs are transparent: the upgrade to higher bit rates and the support of new applications are simpler for a PON than for an active node architecture.

A PON consists of an Optical Line Terminal (OLT), located at the central office and optical nodes which are located in the subscribers' premises and are called Optical Network Units (ONUs) [6]. All ONUs are connected to the OLT through a passive combiner/splitter (Fig. 1). The communication between the ONUs is realized only through the OLT. Signals from the ONUs are combined in the passive element and are transmitted to the OLT through a single fiber; signals from the OLT are separated in the passive element and are then sent to ONUs. In other words, in the downstream direction (from the OLT to the ONUs) traffic is sent from one point to multiple points, while in the upstream direction (from the ONUs to the OLT), traffic from multiple points, reaches only one point.

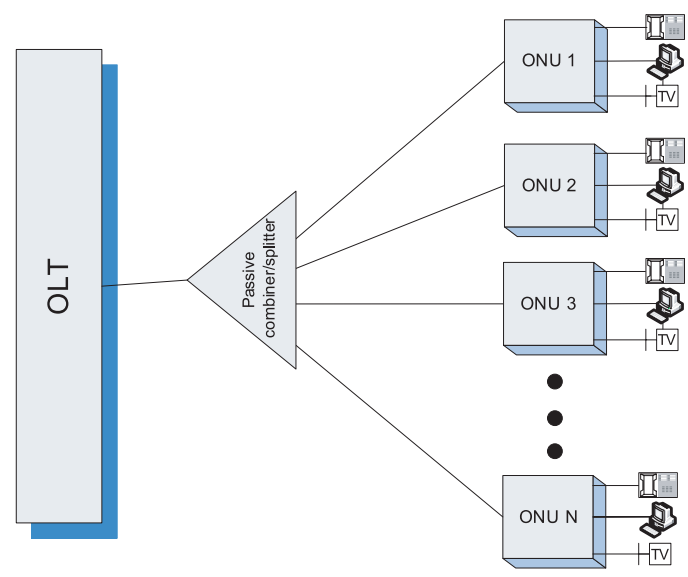

Fig. 1 A typical PON infrastructure

PONs come in different flavors, depending on the multiple access schemes they deploy. TDMA PONs are already standardized and not only are commercially available but also have been deployed in some countries. The APON, i.e. Asynchronous Transfer Mode PON and the Broadband PON (BPON) have been standardized by the International Telecommunications Union - Telecommunication Standardization Sector (ITU-T) (G.983). Likewise, the Gigabit PON (GPON); and the Ethernet PON (EPON) have been standardized by the ITU-T G.984 and IEEE 802.3ah, respectively. Nowadays, although the TDMA-based PON configurations are the most popular configurations for providing Fiber-To-TheHome/Fiber-To-The-Premises (FTTH/FTTP) services, a number of next-generation PON architectures are being studied too, in order to satisfy future bandwidth needs. Dynamic bandwidth allocation is an effective solution for improving the bandwidth utilization in the network. Another solution is the implementation of the Wavelength Division Multiplexing (WDM) technology in the PONs Therefore, PONs based on TDMA can be upgraded, wherein multiple channels can be used both in the upstream and the downstream directions, resulting in TDMA-WDM PONs. The next generation of PONs are the key elements for satisfying the future high bandwidth demands coming from the steadily increasing number of users and from the emerging bandwidth intensive services [7].

In this paper we present a call-level analysis of TDMA PONs and hybrid TDMA-WDM PON configurations for the upstream direction, with dynamic bandwidth allocation. The presented analysis is based on well-established teletraffic models [8], [9], [10]. We calculate Call Blocking Probabilities (CBP) that occur due to the restricted bandwidth of a wavelength. We consider that calls belong to different service-classes with Poisson arrival process. We also study the case where the traffic overflowing the capacity of the primary wavelength is offered to a secondary wavelength.

The rest of this paper is organized as follows. Section 2 provides an overview of the TDMA PONs and the WDM PONs. Section 3 presents the analytical models for calculating the CBP in the aforementioned PON configurations. In section 4 we provide analytical and simulation results of the presented models. We conclude in Section 5.

\section{Multiple Access Techniques in Passive Optical Networks}

\subsection{TDMA Passive Optical Networks}

Current PON configurations are based on Time Division Multiplexing (TDM) technology. The benefits of implementing TDM in PONs are several, such as the low cost of the users' equipment (simple optical transceivers that are tuned to a single frequency) and the flexibility concerning the addition of new users. The first generation of PONs has been standardized by ITU-T G.983 and uses Asynchronous Transfer Mode (ATM) as the Medium Access Control (MAC) protocol. The capacity of the upstream direction is $155.52 \mathrm{Mbps}$, while the capacity of the downstream direction is 155.52 or 622.08 Mbps [11]. The upstream channel operates at $1.3 \mu \mathrm{m}$, whereas the downstream channel operates at $1.5 \mu \mathrm{m}$. The maximum splitting ratio (i.e. the maximum number of ONUs that the network can support) is 32 or 64 and the maximum distance between the OLT and the ONUs is $20 \mathrm{Km}$. A standard singlemode fiber is used in all links i.e., for the connection between the OLT and the passive device and for the connections between the passive device and the ONUs. For the upgrade of the APON, a newer version of the original G.983.1 standard published in 2005 [12] added 1244.16 Mbps downstream transmission rate and $622.08 \mathrm{Mbps}$ upstream transmission rate. Because the term APON gave the impression that only ATM-based services can be supported, ITU-T introduced the term BPON, since these networks can support video and Ethernet traffic. 


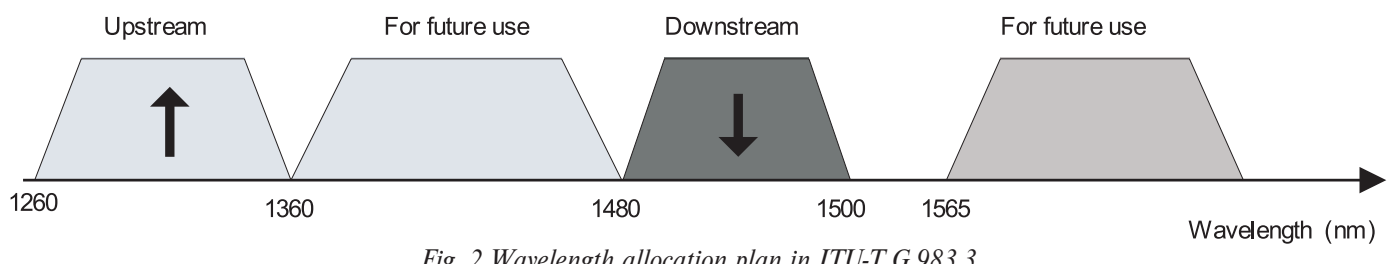

Fig. 2 Wavelength allocation plan in ITU-T G.983.3

The extension of the capacity of PONs to the Gbps arena is realized with the introduction of the G.984.x standard series for the GPON. The GPON architecture is similar to the APON/BPON architecture, whereas the maximum splitting ratio is 128 and the distance between the OLT and the ONUs is up to $30 \mathrm{Km}$. The GPON supports downstream data rates of 1244.16 or 2488.32 Mbps in the wavelength range $1480-1500 \mathrm{~nm}$, while in the upstream direction the GPON supports 155.52, 622.08, 1244.16, or 2488.32 $\mathrm{Mbit} / \mathrm{s}$ data rates, in the wavelength range $1260-1360 \mathrm{~nm}$ [4].

In these TDMA PONs the bandwidth either in the upstream or the downstream direction is allocated by the OLT to the ONUs, based on their bandwidth requests and their Service Level Agreements (SLA). Generally, two are the main algorithms that are used for bandwidth allocation; the Static Bandwidth Allocation (SBA) and the Dynamic Bandwidth Allocation (DBA). Since SBA distributes a fixed bandwidth to each ONU resulting in low utilization of the network's capacity, DBA is an efficient method for a flexible bandwidth allocation and for eliminating idle periods.

Although TDMA PONs provide significant bandwidth boost as compared to copper-based access networks, their capacity will be exhausted as bandwidth-hungry applications such as High Definition TV (HDTV) and video conferencing become available. An upgrading approach for the TDMA-PONs is to combine the TDMA technology together with the WDM technology. In the resulting hybrid TDMA-WDM PON, multiple wavelengths are used in upstream and downstream directions, so that the access network becomes flexible and efficient in providing the required bandwidth to the users [13]. In early TDMA-WDM PONs systems, each ONU uses expensive optical transmitters, generating a unique wavelength. Cost-effective approaches [6], [14] make TDMA-WDM PONs the ideal solution for broadband access networks.

\subsection{TDMA-WDM Passive Optical Networks}

PONs based on TDMA can be upgraded, wherein multiple channels can be used both in the upstream and the downstream directions. The implementation of WDM technology in PONs stumbled at the high cost of the transceivers, which must be tuned in any of the supported wavelengths. New advantages in the area of optical modulation brought forward the idea of using WDM in order to increase the bandwidth capacity. To exploit the vast bandwidth in the optical fiber and make the value out of the capitalintensive fiber plants, ITU-T specified Coarse WDM (CWDM) overlay on the original PON infrastructures [15]. Fig. 2 illustrates the wavelength grid, specified in ITU-T G.983.3. The two bands that are reserved for future use 1) $1360-1480 \mathrm{~nm}$ and 2) $1565 \mathrm{~nm}$ and beyond, may be used by existing fiber plants for supporting new services or overflow traffic.

Another way to increase PON-system scalability is to use Dense WDM (DWDM) [16]. The passive optical splitter/combiner is replaced by an Arrayed Waveguide Grating (AWG) router. Each transmitter-receiver pair (located at the OLT and an ONU or vice versa) is set at a specific wavelength band of a port of the AWG, to which the pair is connected. In this way a single wavelength may be used for the connection of an ONU with the OLT, for the upstream and/or the downstream direction, offering excellent privacy to each ONU. Fig. 3 illustrates a simple WDM PON configuration, where the wavelengths $\lambda 1$ and $\lambda 2$ are used in the upstream direction, while the wavelengths $\lambda 3$ and $\lambda 4$ are used in the downstream direction. Moreover, WDM-PONs provide easy pay-as-you-grow upgrade, since each wavelength may run at different speed, while each subscriber's services can be configured and changed independently from the services of other subscribers.

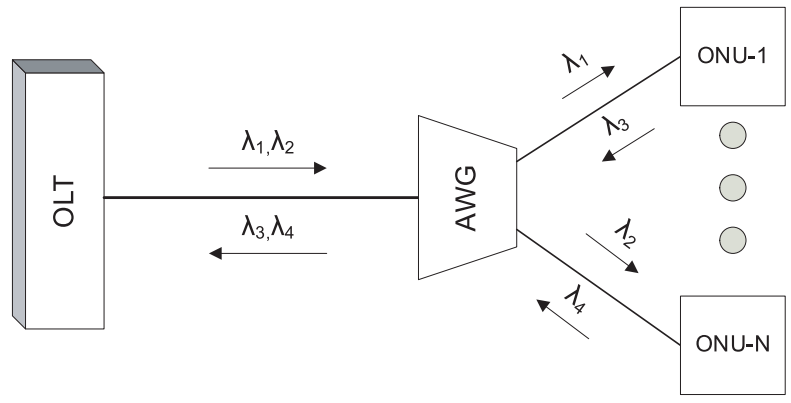

Fig. 3 A typical WDM-PON configuration

For the upgrade of an existing TDMA PON to a hybrid TDMA-WDM PON the fiber plant remains unchanged, while modifications on the ONU's equipment are necessary, in order for each ONU to connect to the OLT using a single wavelength. To make ONUs operational in a WDM PON, they need to be identical, yet capable of functioning according to the wavelength plan. Such ONUs are called "colorless ONUs". The installation of colorless ONUs increases the overall cost, complicates maintenance and decreases the user friendliness. Alternatively, several approaches may be used [17], [18] which can benefit of economy of scale, and thus lower costs. 


\section{Call-level performance analysis of PONs}

\subsection{TDMA PON case}

We consider a TDMA PON (with the configuration illustrated in Fig. 1) with $N$ ONUs. Let $T$ bandwidth units (b.u.) be the capacity of the optical fiber that connects the passive element to the OLT. Calls arrive to each ONU according to a Poisson process and are groomed onto the optical fiber. Each ONU accommodates $K$ service-classes. Each service-class $k(k=1, \ldots, K)$ call requires $b_{k}$ b.u. from the OLT, in order to be serviced (fixed bandwidth requirement). The arrival rate of service-class $k$ calls is denoted by $\lambda_{k}$, while their service times are exponentially distributed with mean $\mu_{k}^{-1}$. Considering that the total offered traffic-load of serviceclass $k$ is $a_{k}=N \cdot \lambda_{k} \mu_{k}^{-1}$, the occupancy distribution $q(i)$ of the optical fiber is given by the following recurrent formula (Kaufman/Roberts recursion, [8], [9]):

$$
i q(i)=\sum_{k=1}^{K} a_{k} b_{k} q\left(i-b_{k}\right) \quad \text { for } \quad i=1, \ldots, T
$$

with $q(i)=0$ for $i<0$ and $\sum_{i=0}^{T} q(i)=1$.

The CBP of service-class $k$ calls can be calculated given that the blocking states for a service-class $k$ call are the last $b_{k}$ states (states with occupancies greater than $T-b k$ ). Therefore:

$$
B_{k}=\sum_{i=T-b_{k}+1}^{T} q(i)
$$

Let us consider that the aforementioned PON configuration can additionally support one of the two bands available for the future use that are indicated in Fig. 2. This wavelength band corresponds to a secondary capacity (denoted by $T_{2}$ ), and calls that are lost in the primary wavelength band (with capacity denoted by $T_{1}$ ) are offered to this secondary wavelength. In order to calculate the traffic loss in this hierarchical network, we follow the analysis of [10], where the Multiservice Overflow Approximation (MOA) method is proposed. The traffic load of service-class $k$ offered to the primary capacity is denoted by $a_{k}^{0}$ and the bandwidth requirement of a service-class $k$ call is denoted by $b_{k}$. The offered overflow traffic of service-class $k$ is described by its mean value, denoted by $a_{k}^{1}$ and its peakedness, denoted by $z_{k}^{1}$.

The MOA method includes three steps. In the first step we calculate the blocking probabilities of service-class $k$, denoted by $B_{k}^{0}$ using (2). In the second step we calculate the mean and the peakedness of the service-class $k$ overflow traffic from the primary to the secondary wavelength. The mean of the overflow traffic is given by the average traffic loss in the primary capacity, therefore:

$$
a_{k}^{1}=a_{k}^{0} \cdot B_{k}^{0}
$$

For the calculation of the peakedness of the overflow traffic from the primary wavelength, we map this primary capacity into an "imaginary" overflow model, where the traffic streams which are originally offered the primary capacity are assumed to direct to K "imaginary" bandwidth groups, classified by service-classes. We denote by $\beta_{k}^{0}$ the number of "imaginary" bandwidth groups for service-class $k$. The bandwidth requirement of each serviceclass $k$ call in the "imaginary" bandwidth groups is assumed to be $d_{k}^{*}=1$. In order to calculate $\beta_{k}^{0}$, we use the function

$$
E\left(\beta_{k}^{0}, a_{k}^{0}\right)=B_{k}^{0}
$$

where $E()$ denotes the loss probability of the Erlang loss function. The peakedness $z_{k}^{*}$ of service-class $k$ overflow traffic from the "imaginary” bandwidth groups $\beta_{k}^{0}$ can be calculated by Riordan's equation [10]:

$$
z_{k}^{*}=1-a_{k}^{*}+\frac{a_{k}^{0}}{1+\beta_{k}^{0}-a_{k}^{0}+a_{k}^{*}}
$$

where $a_{k}^{*}$ is the mean of service-class $k$ overflow traffic from the "imaginary" $\beta_{k}^{0}$ bandwidth groups. Finally, in the third step we obtain the characteristics of the service-class $k$ overflow traffic from the primary wavelength, whereas the mean traffic is given by (3) and the peakedness is given by:

$$
z_{k}^{1}=z_{k}^{*} \cdot z_{k}^{0}=z_{k}^{*}
$$

since $z_{k}^{0}=1$ is the peakedness of the Poisson traffic originally offered to the primary wavelength. The CBP of service-class $k$, denoted by $B_{k}^{1}$, can be calculated by a modification of the Fredericks \& Hayward's approximation method [10], by using (1) and (2), where the capacity $T$ is equal to $T_{2} / z_{k}^{1}$ and the service-class $k$ offered traffic load $a_{k}$ is equal to $a_{k}^{1} / z_{k}^{1}$.

\subsection{TDMA-WDM PON case}

The aforementioned analysis can be extended in order to provide the CBP in a TDMA-WDM PON. Since each one of the $N$ ONUs utilizes a different wavelength, the distribution of the occupied b.u. inside the wavelength $n(n=1, \ldots, N)$ is given by:

$$
i q_{n}(i)=\sum_{k=1}^{K_{n}} a_{k, n} b_{k, n} q\left(i-b_{k, n}\right) \quad \text { for } \quad i=1, \ldots, T
$$

with $q_{n}(i)$ for $i<0$ and $\sum_{i=0}^{T_{n}} q_{n}(i)=1$.

where the $n^{\text {th }}$ ONU utilizes a wavelength with bandwidth capacity $T_{n}$ and supports $K_{n}$ service classes, while is the offered traffic-load and $b_{k, n}$, is the bandwidth requirement of service-class $k_{n}\left(k_{n}=1\right.$, ..., $K_{n}$ ) calls. The CBP of service-class $k_{n}$ calls can be calculated by

$$
B_{k, n}=\sum_{i=T_{k}-b_{k, n}+1}^{T} q_{n}(i)
$$

It is evident that the MOA method can be used for the case that the PON supports additional wavelengths for carrying the overflow traffic from the primary wavelengths. 


\section{Numerical Results}

In this section we provide analytical and simulation CBP results for the two PON configurations presented in this work, by providing two examples. In the first example we consider a TDMA PON with capacity $T$, while the number of ONUs is 32 . The TDMA PON accommodates a data service and a video service with bandwidth requirements 20 b.u. and 6 b.u., respectively. We assume that 1 b.u. corresponds to $1 \mathrm{Mbps}$, therefore the capacity $T$ is 155 , 622,1244 or 2048 , following the upstream data rates of the TDMA PONs. We assume that both service-classes offer the same amount of traffic, therefore $a_{1}=a_{2}=a$. Fig. 4(a)-(d) provide analytical and simulation CBP results versus the traffic-load that is offered by each ONU. The comparison of the analytical and the corresponding simulation results show a completely satisfactory accuracy of the proposed model. The study of these figures reveals the maximum offered traffic-load that each PON configuration can support, for specific values of blocking. In the second example we consider that calls that are lost in the primary capacity are offered to a secondary wavelength with the same capacity $T$. Fig. 5 (a)-(d) illustrate the analytical and simulation CBP results in the secondary wavelength versus the traffic-load $a$ that is offered by each ONU.
All results show that the model's accuracy is completely satisfactory.

\section{Conclusion}

In conclusion, we present a call-level performance analysis of different PON configurations, for Poisson traffic. We study the upstream direction of TDMA PONs and TDMA-WDM PONs. The models are evaluated through extensive simulation in respect of their results. The accuracy of the models was found to be absolutely satisfactory. The presented models may help network operators to obtain full knowledge of the network's behaviour under extremely high traffic-loads. Moreover, these models may provide useful information to service providers before installing a new PON, by indicating the network parameters for optimum performance. Therefore in our future work we shall continue to study the performance of PONs, by taking into account finite number of traffic sources, while we will include in our study not only the upstream but also the downstream direction (from OLT to the ONUs).
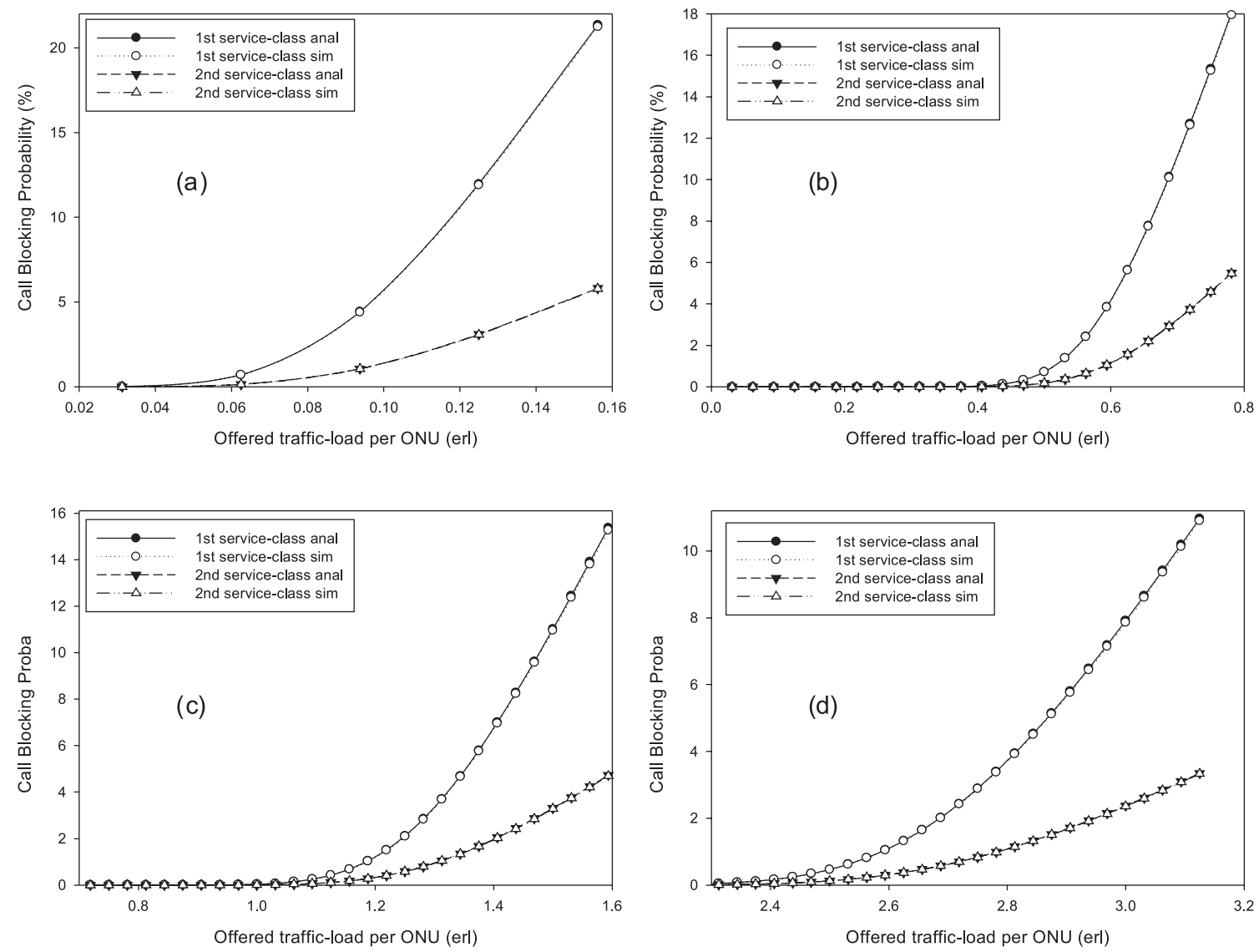

Fig. 4 Analytical and simulation CBP results for 4 different values of the optical fiber capacity 

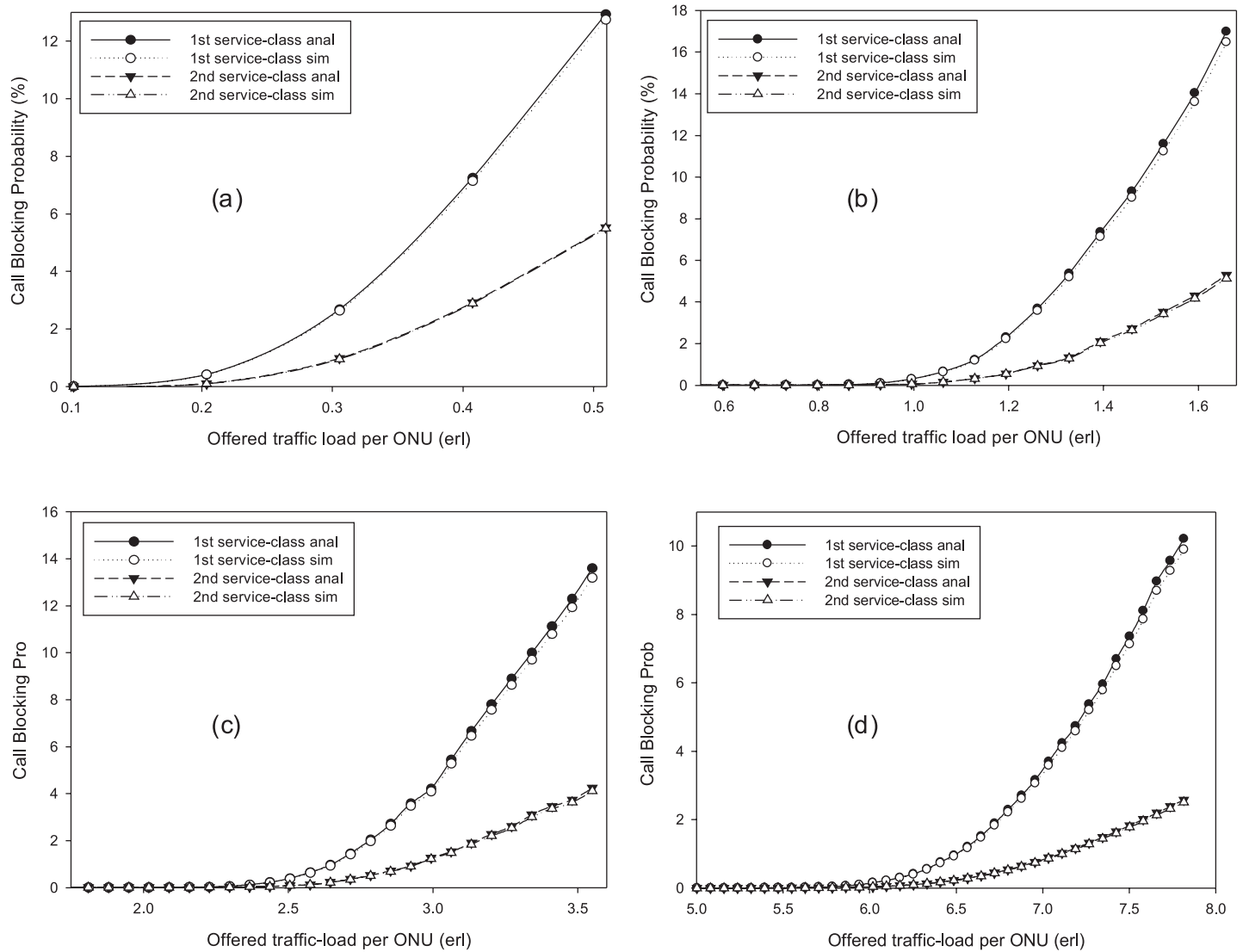

Fig. 5 Analytical and simulation CBP results for the secondary wavelength for 4 different values of the wavelength capacity

\section{References}

[1] MUKHERJEE, B.: Optical WDM Networks, Springer, 2006.

[2] SIVAliNGAM, K. M., SUBRAMANIAM, S.: Emerging Optical Network Technologies, Springer Science, 2005.

[3] KRAMER, G.: Ethernet Passive Optical Networks, McGraw Hill, 2005.

[4] KOONEN, T.: Fiber to the Home/Fiber to the Premises: What, Where, and When?, Proc. of the IEEE, Vol. 94, No. 5, pp. 911-934, May 2006.

[5] LIN, C.: Broadband Optical Access Networks and Fiber-to-the-Home, Wiley, 2006.

[6] EFFENBERGER, F., CLEARY, D., HARAN, O., KRAMER, G., DING LI, R., ORON, M., PFEIFFER, T.: An Introduction to PON Technologies, IEEE Communications Magazine, pp. S17-S25, March 2007.

[7] DAVEY, R., BOURGART, F., MCCAMMON, K.: Options for Future Optical Access Networks, IEEE Communications Magazine, October, 2006.

[8] KAUFMAN, J. S.: Blocking in a shared resource environment, IEEE Transactions on Communications, 10/1981, pp.1474-1481.

[9] ROBERTS, J. W.: A service system with heterogeneous user requirements, G. Pujolle (Ed.), Performance on Data Communication Systems and their Applications, North Holland, Amsterdam, 1981, pp. 423-431.

[10] HUANG, Q., KO, K-T., IVERSEN, V. B.: An Approximation Method for Multiservice Loss Performance in Hierarchical Networks, L. Mason, T. Drwiega, and J. Yan (Eds.): ITC 2007, LNCS 4516, pp. 901-912.

[11] UEDA, H., OKADA, K., FORD, B., MAHONY, G., HORNUNG, S., FAULKNER, D., ABIVEN, J., DUREL, S., BALLART, R., ERICKSON, J.: Deployment status and common technical specifications for a B-PON system, IEEE Communication Magazine, 12/2001, pp. 134-141.

[12] ITU-T Recommendation G.983.1, Broadband Optical Access Systems Based on Passive Optical Networks (PON), 2005. 


\section{COMMNICOIIONS}

[13] DHAINI, A. R., ASSI, C. M., MAIER, M., SHAMI, A.: Dynamic Wavelength and Bandwidth Allocation in Hybrid TDM/WDM EPON Networks, IEEE/OSA Journal of Lightwave Technology, 1/2007, pp. 277-286.

[14] GUTIERREZ, D., SHAW, W.-T., AN, F.-T., KIM, K. S., HSUEH, Y.-L., ROGGE, M., WONG, G., KAZOVSKY, L. G.: Next generation optical access networks, (Invited Paper), Proc. of IEEE/Create-Net BroadNets, Oct. 2006.

[15] LAM, C.: Passive Optical Networks, Principles and Practise, Academic Press, 2007.

[16] GROBE, K., ELBERS, J-P.: PON in Adolescence: From TDMA to WDM-PON, IEEE Communications Magazine, pp. 26-34 January 2008

[17] FRIGO, N. J., IANNONE, P., REICHMANN, K. C.: Spectral slicing in WDM passive optical networks for local access", in Proc. ECOC 1998, pp. 119-120.

[18] FRIGO, N. J., IANNONE, P. P., REICHMANN, K. C., WALKER, J. A., GOOSSEN, K. W., ARNEY, S. C., MURPHY, E. J., OTA, Y., SWARTZ, R. G.: Demonstration of performance-tiered modulators in a WDM PON with a single shared source, Proc. of ECOC 1995, pp. 441-444. 